\title{
Evaluación de la aplicación del programa educativo para el entrenamiento de los estudiantes que participan en las Olimpiadas de Química
}

Nilda Delgado-Yanes ${ }^{1}$

Universidad de las Ciencias Informáticas - Cuba nildady@uci.cu

Alipio Omar Pérez-Jacinto ${ }^{2}$

Universidad de Artemisa - Cuba

opejota@gmail.com

DOI: $\underline{\text { https://doi.org/10.21158/2357514x.v7.n2.2019.2528 }}$

Cómo citar este artículo: Delgado-Yanes, N.; Pérez-Jacinto, A. O. (2019). Evaluación de la aplicación del programa educativo para el entrenamiento de los estudiantes que participan en las Olimpiadas de Química. Revista Virtu@Imente, 7(2), 47-72. DOI: https://doi.org/10.21158/2357514x.v7.n2.2019.2528

Fecha de recepción: 03 de abril 2019

Fecha de aprobación: 02 de noviembre de 2019
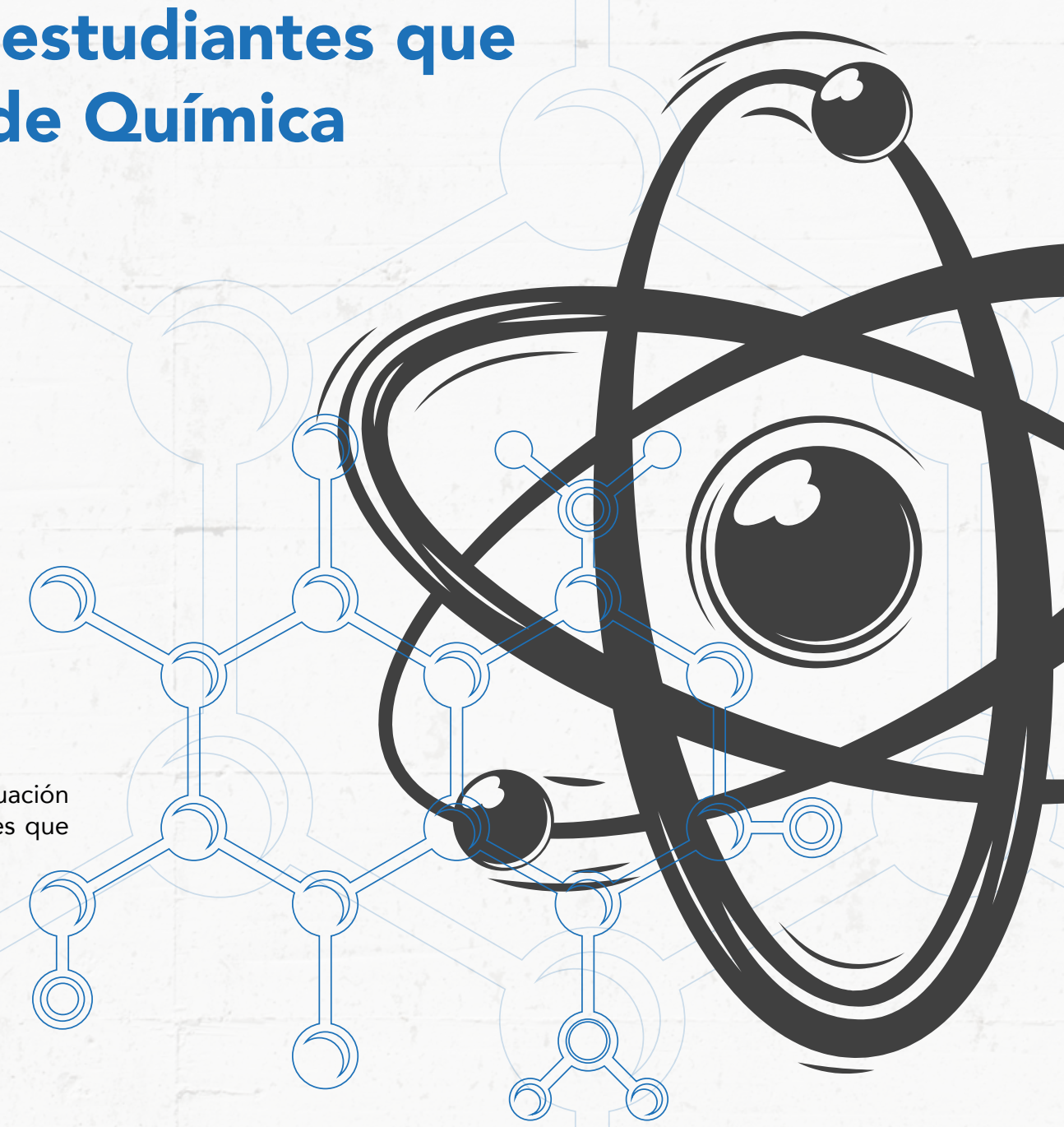

${ }_{1}^{1}$ Doctora en Ciencias Pedagógicas. En el área de investigación ha participado en proyectos relacionados con el desarrollo del talento académico y sobre Pedagogía y Didáctica General. Actualmente forma parte del proyecto de investigación «Modelo pedagógico adecuado a las demandas en la formación de profesionales del siglo XXl» de la Universidad de Artemisa.

ORCID: https://orcid.org/0000-0002-3617-7719

${ }^{2}$ Doctor en Ciencias Pedagógicas. En el área de investigación ha participado en proyectos relacionados con estudios teóricos sobre la Estadística Inferencial.

ORCID: https://orcid.org/0000-0002-1485-7692 


\section{RESUMEN}

La educación en Cuba ha prestado gran atención al entrenamiento de los estudiantes que participan en los concursos de conocimientos y en las olimpiadas. Por esta razón, los estudiantes ganadores de la Olimpiada Nacional realizan un proceso de preparación especial, con el fin de participar en los eventos internacionales. El proceso que se desarrolla constituye una de las vías de atención al talento académico en química, pues la mayoría de ellos optan por carreras relacionadas con esta ciencia. Esto justifica el objetivo de este trabajo: evaluar el programa educativo aplicado a los estudiantes que participaron en el proceso de entrenamiento para su participación en las Olimpiadas de Conocimientos de Química, desde las esferas cognitiva-instrumental, motivacional-afectiva y psicosocial, aspectos que forman parte de una investigación realizada. Los métodos investigativos utilizados a fin de cumplir este objetivo fueron: análisis documental, análisis y síntesis, la prueba estadística, determinación del coeficiente de correlación multidimensional, técnicas y pruebas psicológicas. Como resultado se presentan las acciones a realizar para la ejecución del programa, la información obtenida luego de la aplicación de varios instrumentos y la utilización del coeficiente multidimensional en la determinación de la coherencia entre los indicadores propuestos en las tres dimensiones analizadas durante la investigación.

Palabras clave: enseñanza de ciencias; enseñanza de química; competencias en ciencias; esfera cognitiva-instrumental; esfera motivacional-afectiva; esfera psicosocial. 


\section{Evaluation of the implementation of the educational program for the training of students participating in the Chemistry Olympiad}

\section{ABSTRACT}

Education in Cuba has paid great attention to the training of students who are participating in knowledge competitions and the Olympics. For this reason, the students who win the National Olympics undergo a special preparation process in order to participate in international events. The process that has been developed constitutes one of the ways of showing attention to the academic talent in chemistry, since most of the students choose careers that are related to this science. This is what justifies the objective of this work: to evaluate the educational program that is applied to the students who participated in the training process for their participation in the Knowledge of Chemistry Olympiad, from the cognitiveinstrumental, motivational-affective, and psychosocial spheres, aspects that are part of research undertaken. The research methods that were used in order to fulfill this objective were: documentary analysis, analysis and synthesis, statistical test, determination of the multidimensional correlation coefficient, techniques, and psychological tests. As a result, this document shows the actions that must be carried out for the implementation of the program, the information that was obtained after the application of various instruments and the use of the multidimensional coefficient in determining the consistency among the indicators that were proposed in the three dimensions analyzed during the investigation.

Keywords: science teaching; chemistry teaching; science competencies; cognitive-instrumental sphere; motivational-affective sphere; psychosocial sphere. 


\section{Avaliação da aplicação do programa educacional para a formação dos alunos participantes das Olimpíadas de Química}

\section{RESUMO}

A educação em Cuba prestou muita atenção ao treinamento de estudantes que participam de concursos de conhecimento e das Olimpíadas. Por esse motivo, os alunos vencedores da Olimpíada Nacional realizam um processo de preparação especial, a fim de participar de eventos internacionais. O processo desenvolvido constitui um dos canais de atenção ao talento acadêmico em química, pois a maioria deles escolhe carreiras relacionadas a essa ciência. Isso justifica o objetivo deste trabalho: avaliar o programa educacional aplicado aos alunos que participaram do processo de treinamento para sua participação nas Olimpíadas do Conhecimento de Química, nas esferas cognitivo-instrumental, motivacional-afetiva e psicossocial, aspectos que formam parte de uma investigação. Os métodos de pesquisa utilizados para atingir esse objetivo foram: análise documental, análise e síntese, teste estatístico, determinação do coeficiente de correlação multidimensional, técnicas e testes psicológicos. Como resultado, são apresentadas as ações a serem executadas para a execução do programa, as informações obtidas após a aplicação de vários instrumentos e o uso do coeficiente multidimensional na determinação da coerência entre os indicadores propostos nas três dimensões analisados durante a investigação.

Palavras-chave: ensino de ciências; ensino de química; competências científicas; esfera instrumentalcognitiva; esfera motivacional-afetiva; esfera psicossocial. 


\title{
Évaluation du programme éducatif de formation des étudiants participant aux olimpiades de la chimie
}

\section{RÉSUMÉ}

\begin{abstract}
Le systéme éducatif de Cuba accorde une grande attention à la formation des étudiants participant à des concours de connaissances. Pour cette raison, les étudiants vainqueurs des concours nationaux réalisent un processus de préparation spécial pour participer à des événements internationaux. Le processus mis en place propulse les jeunes talents en chimie dont la plupart choisiront des cursus scientifiques. L'objectif de ce travail d'investigation est d'évaluer le programme éducatif suivi par les étudiants ayant participé au processus de formation et de participation aux Olympiades de la Connaissance en Chimie. Nous nous intéresserons aux sphères cognitivo-instrumentales, motivationnelle-affective et psychosociale qui sont les aspects structurant le cadre de notre travail. Les méthodes d'investigation de cette recherche ont été: l'analyse documentaire, analyse de synthèses, les preuves statistiques, la détermination du coefficient de corrélation multidimentionnelle, des techniques et examen psychologiques. Les résultats présentent des actions à mener pour la culmination du programme, les informations obtenues après l'application de plusieurs instruments ainsi que l'utilisation du coefficient multidimentionnel pour déterminer la cohérence des indicateurs proposés lors des trois dimensions principales analyses de cette recherche.
\end{abstract}

Mots-clés: enseignement des sciences, enseignement de la chimie, compétences en sciences, sphère cognitivo-instrumentale, sphère affectivo-motivationnelle, sphère psychosociale. 


\section{Introducción}

El entrenamiento de los estudiantes orientado a los concursos y las olimpiadas de conocimientos es una de las vías en la preparación de los que optan por carreras como Licenciatura en Química, en cuanto ciencia básica de gran importancia para el país. Las transformaciones realizadas en la educación preuniversitaria con el fin de lograr una orientación profesional que responda al momento histórico que vive la sociedad tiene como prioridad la atención de los estudiantes que sienten inclinación por el estudio de esta asignatura.

Los concursos de conocimientos en Cuba comenzaron a desarrollarse desde los primeros años de la Revolución, con el objetivo de incentivar a los estudiantes y a los profesores en el estudio, así como a profundizar en las asignaturas por las cuales se competía; más tarde comenzó la participación en las olimpiadas de conocimientos y habilidades a nivel internacional.

En la asignatura de química se compite desde 1985, cuando se recibió la invitación a la $17^{\mathrm{a}}$ Olimpiada Internacional de Química, realizada en Bratislava, en la República Socialista de Checoslovaquia. En la actualidad, se presentan estudiantes en estos eventos y en las olimpiadas iberoamericanas y centroamericanas.
Los estudiantes que compiten en esos eventos se seleccionan de un grupo de estudiantes de educación preuniversitaria, quienes integran la Preselección Nacional de Química y se escogen de los ganadores en los concursos nacionales de conocimientos.

Los programas que se han seguido en el entrenamiento de los estudiantes, en un inicio, tenían naturaleza cognitiva, con el fin principal de obtener altos resultados académicos en las competencias. Con el paso de los años comenzó a tenerse en cuenta, además de la esfera cognitiva-instrumental, la motivacional-afectiva y psicosocial, con el propósito de lograr una preparación integral que les permita enfrentar los retos futuros de la sociedad, pero sin una planificación y sin la participación generalizada de la familia y de instituciones científicas.

A fin de cumplir ese objetivo, y luego del estudio de la bibliografía consultada y la exploración realizada en la práctica pedagógica en el proceso de entrenamiento de los estudiantes de la Preselección Nacional, se plantea como objetivo general de esta investigación evaluar el programa educativo aplicado a los estudiantes que participaron en el proceso de entrenamiento para su participación en las Olimpiadas de Conocimientos de Química, desde las esferas cognitiva-instrumental, motivacional-afectiva y psicosocial. Los objetivos específicos de la investigación se indican en el apartado de la presentación del programa educativo. 


\section{Referentes teóricos}

En el resultado de la investigación realizada en el proyecto «Modelo de Intervención Educativa para el Desarrollo del Talento», se expresa la concepción cubana acerca del desarrollo del talento, la cual concreta, en el 2005, un colectivo de autores de la Universidad de Ciencias Pedagógicas «Enrique José Varona» y de otras universidades, en el que se asume una concepción teórica y metodológica basada en una educación desarrolladora y se entiende el talento desde una perspectiva que resalta su naturaleza dialéctica, contextualizada e interactiva. En la Plataforma Programática de la mencionada investigación se asume el talento como «una configuración, es el resultado de una integración dinámica -estructural y funcional- de un conjunto heterogéneo y variable de procesos, cualidades y formaciones psicológicas» (Castellanos, 2005, p. 21).

Los autores de la plataforma citada en el párrafo anterior asumen esta definición por considerar el talento una configuración de la personalidad, la cual se estructura en función de una serie de condiciones internas y externas que intervienen de forma recíproca; un potencial que necesita estimularse mediante intervenciones educativas que tengan en cuenta la atención al desarrollo en los sujetos de las capacidades cognitivas, afectivas y sociales. Esta concepción la asumen los autores de la investigación.

Un concepto subordinado al anterior sería el de talento académico, referido a los sujetos que «se distinguen por sus altas potencialidades y/o capacidades actualizadas en forma de desempeños creativos o sobresalientes en una o varias áreas del conocimiento y/o actividad que conforman el currículo de los diferentes niveles de enseñanza» (Castellanos, 2003 , p. 21). En este caso se encuentran los estudiantes con altas potencialidades y un desempeño exitoso en el área de la química.

Los autores de este trabajo consideran el talento académico en química como una configuración y un resultado de procesos, cualidades y formaciones psicológicas en los que se articula el desarrollo de las capacidades que permiten la solución de problemas en el área de la química, con otros procesos psíquicos que tienen la función de movilizar, orientar y sostener al sujeto en esa área y tener desempeños valiosos para la sociedad (Delgado, 2010).

Al tener en cuenta la definición de talento asumida por los autores y considerar que el talento representa la unidad dinámica de componentes complejos de la personalidad, tales como las capacidades generales y especiales, la creatividad y la motivación, en interacción con la sociedad mediante la actividad, se acota que pueden darse diferentes tipos de talento entre los que se encuentra el académico y, de manera específica, el talento académico en química.

En el proceso de formación y desarrollo del sujeto dirigido a insertarse en una sociedad determinada, desempeña una función fundamental la educación y, dentro de esta, la atención al talento, como parte de su preparación para 
la vida en sociedad; no obstante, no siempre se tiene en cuenta este aspecto por parte de las diferentes naciones.

En Cuba, una de las principales prioridades es que todas las personas reciban una educación de calidad y una de las esferas de esta es la educación del talento -atención al talento-, la cual,

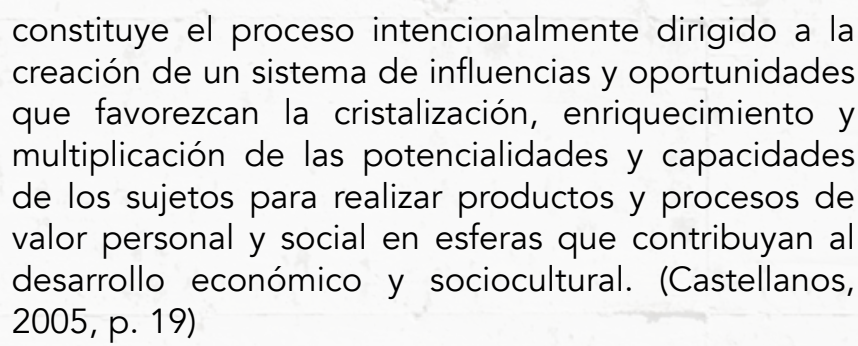

La atención al talento se lleva a cabo mediante tres tareas básicas: la identificación, la estimulación y la orientación.

La identificación «es concebida como un componente intrínseco del proceso de perfeccionamiento educativo» (Castellanos, 2005, p. 24 ); a juicio de sus autores, «supone todo el proceso de detección/reconocimiento de los sujetos talentosos o potencialmente talentosos y su diagnóstico más profundo con vistas a decisiones interventivas» (Castellanos, 1997, p. 11), en el cual el docente evalúa las fortalezas y las necesidades educativas de los estudiantes con la finalidad de proyectar estrategias y programas que faciliten el desarrollo de las potencialidades talentosas.

Por su parte, la estimulación se piensa «como un término genérico que engloba la diversidad de iniciativas que, desde la propia escuela, pueden estructurarse para contribuir al desarrollo pleno en cada estudiante, de sus potencialidades y capacidades, y muy en especial, de sus talentos» (Castellanos, 2005, p. 28). Las iniciativas se conciben en forma de estrategias, programas y proyectos dirigidos a desarrollar y promover los talentos.

La orientación del talento, como otra de las tareas básicas de atención al talento, en correspondencia con el proceso de enriquecimiento,

\section{comprende el despliegue de una ayuda, guía y consejo profesional-especializado a los sujetos talentosos, a sus familias y personas cercanas, que tenga como centro las necesidades y problemas socioafectivos que ellos pueden enfrentar, con vistas a promover su desarrollo personal y social óptimo. (Castellanos, 1997, p. 11)}

En este campo se contempla al propio estudiante, a sus familias, a los maestros y a cualquier otra persona que requiera una asesoría en la comprensión del tema.

En esta tarea se deben tener en cuenta las necesidades educativas y los problemas socioafectivos que presentan los estudiantes talentosos y la dirige el profesor, en su función de orientador del proceso de enseñanza-aprendizaje. La orientación a la familia puede llevarse a cabo en las reuniones de padres o en entrevistas personales con ellos, a fin de lograr la mejor comprensión de su hijo y despertar la necesidad de su atención diferenciada.

Las alternativas que se utilizan en la atención educativa al talento se centran en dos grandes grupos de factores: la organización de las opciones educativas y los cambios en el currículo. 
En Cuba, las alternativas más utilizadas en la organización que se le da a las opciones educativas es la integración a la escuela y, en algunas ocasiones, en aula común, así como en las adaptaciones curriculares, la compactación curricular y actividades extracurriculares de enriquecimiento tales como los círculos de interés, las clases especiales, los encuentros de monitores, los concursos de conocimientos y habilidades o las sociedades científicas estudiantiles.

En la educación preuniversitaria se utiliza la alternativa de adaptación curricular, principalmente en los institutos preuniversitarios vocacionales de ciencias exactas, donde se atienden de forma diferenciada a los estudiantes con un mayor desarrollo del talento, quienes se preparan para participar en las olimpiadas internacionales de matemática, física, informática y química.

Por otra parte, se considera que las tareas básicas en la educación del talento y las alternativas que se siguen en su atención pueden utilizarse en el desarrollo del talento $y$, en específico, del talento académico en química de los estudiantes que se preparan con el objetivo de participar en los concursos de conocimientos de la asignatura. Para esto se instrumentó un programa educativo, el cual se asumió como,

el plan que abarca un conjunto de actividades con fines educativos, dirigidas al desarrollo del talento académico en química, desde lo cognitivo, motivacional-afectivo y psicosocial, donde se compromete a entrenadores y estudiantes para alcanzar altos niveles en la preparación para su participación en los concursos. (Delgado, 2010)

Los resultados obtenidos en las indagaciones empíricas y teóricas, así como la consulta a los profesores entrenadores en relación con el desarrollo del talento académico en química de los estudiantes de la Preselección Nacional, luego de determinar las dimensiones e indicadores de la variable desarrollo del talento académico en química, permitieron prescribir la estructuración del programa educativo en función de elevar los resultados en los eventos y transformar conductas a seguir en el futuro.

\section{Metodología}

La investigación se desarrolló con un enfoque cuantitativocualitativo, a partir de la aplicación de un programa educativo que contemplara acciones en la esfera cognitivainstrumental, motivacional-afectiva y el psicosocial. Durante su aplicación en la valoración de los resultados se utilizó el coeficiente de correlación multidimensional $r_{p j}$ como técnica de investigación. El coeficiente de correlación multidimensional $r_{p j}$ se considera un procedimiento lógico aceptado por la teoría clásica de los test. 
En el caso que se estudia se utilizaron los siguientes métodos de investigación: analítico-sintético, análisis documental, técnicas y pruebas psicológicas. Además, a partir del empleo del coeficiente de correlación multidimensional $r_{\mathrm{pj}}$ mediante la aplicación del software de procesamiento Cohaerentîa y el procedimiento lógico de ruptura con la racionalidad y la lógica clásicas, con el propósito de determinar la fiabilidad, el grado de coherencia y la objetividad de los resultados.

El grupo de estudio estuvo integrado por 12 estudiantes de la Preselección Nacional de Química, en su Centro de Entrenamiento, con vistas a participar en las olimpiadas de conocimientos de química.

Tabla 1. Dimensión motivacional-afectiva

\begin{tabular}{|c|c|}
\hline \multirow{2}{*}{ Dimensión } & Indicadores \\
\hline \multirow{3}{*}{ Motivacional-afectiva } & Autoestima \\
\cline { 2 - 2 } & Autoconcepto académico \\
\cline { 2 - 2 } & Autoconcepto afectivo \\
\cline { 2 - 2 } & Ansiedad de estado \\
\hline
\end{tabular}

Fuente. Elaboración propia.

Tabla 2. Dimensión psicosocial

\begin{tabular}{|c|c|}
\hline Dimensión & $\begin{array}{c}\text { Indicadores } \\
\text { Satisfacción grupal }\end{array}$ \\
\hline \multirow{3}{*}{ Psicosocial } & $\begin{array}{c}\text { Autoconcepto social } \\
\text {-comunicación con los demás- }\end{array}$ \\
\cline { 2 - 2 } & Apoyo familiar \\
\hline
\end{tabular}

Fuente. Elaboración propia.

La revisión bibliográfica permitió definir algunos conceptos sobre el desarrollo del talento académico. Luego, a partir de estos se procedió a realizar la operacionalización de la variable e identificar las dimensiones e indicadores que expresan, con la mayor objetividad posible, los rasgos distintivos de la variable de estudio concebida como talento académico en química, los cuales se expresan en las tablas 1,2 y 3 . 
Tabla 3. Dimensión cognitiva-instrumental

\begin{tabular}{|c|c|c|}
\hline Dimensiones & Subdimensiones & Indicadores \\
\hline \multirow{18}{*}{ Cognitiva-instrumental } & \multirow{4}{*}{ Conocimientos químicos sobre } & Química general e inorgánica \\
\hline & & Química física \\
\hline & & Química orgánica \\
\hline & & Química analítica \\
\hline & \multirow{12}{*}{ Desarrollo de habilidades en } & Nomenclatura química \\
\hline & & Notación química \\
\hline & & Escritura de ecuaciones \\
\hline & & Interpretación de gráficos \\
\hline & & Interpretación de tablas \\
\hline & & Interpretación cuantitativa de ecuaciones químicas \\
\hline & & Interpretación cualitativa de ecuaciones químicas \\
\hline & & Resolución de problemas de cálculo \\
\hline & & Interpretación espacial de estructuras moleculares \\
\hline & & Montaje de aparatos \\
\hline & & Manipulación de la dotación de laboratorio \\
\hline & & Resolución de problemas experimentales \\
\hline & \multirow{2}{*}{$\begin{array}{l}\text { Estado de procesos psíquicos } \\
\text { cognitivos }\end{array}$} & Razonamiento verbal y flexibilidad del pensamiento \\
\hline & & Razonamiento lógico \\
\hline
\end{tabular}

Fuente. Elaboración propia.

El programa educativo constó de cuatro etapas, las cuales se describen a continuación.

\subsection{Etapa 1}

Diagnóstico del desarrollo del talento académico en química de los estudiantes, en las esferas cognitiva-instrumental, motivacional-afectiva y psicosocial. 
Los objetivos específicos de esta etapa fueron:

- Caracterizar desde una concepción multidimensional el estado inicial del desarrollo del talento académico en química alcanzado por los estudiantes de la preselección.

- Instrumentar e interpretar técnicas de identificación de estudiantes talentosos.

En esta etapa se realizó una reunión metodológica con los profesores que cumplen el rol de entrenadores a fin de valorar la importancia de la caracterización de los estudiantes y de la aplicación del programa educativo en el desarrollo del entrenamiento. Además, se explicó la instrumentación de técnicas de identificación de los estudiantes talentosos.

Una vez se aplicaron las técnicas se procedió al procesamiento de la información y a la interpretación de los resultados, cuyo producto final fue la caracterización de cada estudiante, discutida con los profesores entrenadores.

\subsection{Etapa 2}

Planificación de las acciones de estimulación y orientación para el desarrollo del talento académico en química, en las tres esferas: cognitiva-instrumental, motivacional-afectiva y psicosocial.
Los objetivos específicos de esta etapa fueron:

- Determinar las acciones de estimulación y orientación a realizar para el desarrollo del talento académico en química, en las tres esferas: cognitiva-instrumental, motivacional-afectiva y psicosocial.

- Coordinar las actividades de estimulación y orientación de los estudiantes de la preselección.

Una vez caracterizados los estudiantes, se procedió a determinar las acciones a realizar en el plan de entrenamiento, el cual contenía una fundamentación, los objetivos generales, el sistema de habilidades, el plan temático, el sistema de conocimientos, el sistema de evaluación y la bibliografía.

El plan temático propuesto constó de los temas siguientes:

- Tema 1: contenidos relacionados con química general e inorgánica.

- Tema 2: contenidos relacionados con química analítica.

- Tema 3: contenidos relacionados con química física.

- Tema 4: contenidos relacionados con química orgánica.

- Tema 5: contenidos experimentales. 
Este plan temático se adecuó a los contenidos orientados por el comité organizador del país sede de la olimpiada. También, como parte de esta actividad y en conformidad con la experiencia de los entrenadores, se reflexionó acerca de aquellos contenidos y habilidades que con regularidad han presentado mayores dificultades para los estudiantes y se tomaron decisiones con miras al nuevo periodo de entrenamiento.

Un aspecto que se introdujo en el programa educativo propuesto fue sensibilizar a los entrenadores con la necesidad de desarrollar en sus clases no solo la esfera cognitiva-instrumental, priorizada en entrenamientos anteriores, sino también estimular las esferas motivacionalafectiva y psicosocial, en las cuales se habían constatado insuficiencias en los estudiantes de la preselección. Las actividades propuestas respondieron a realizar tareas que llevaran al razonamiento lógico de los estudiantes y a la relación de los contenidos con la vida cotidiana; para esto se utilizaron medios de enseñanza en soporte digital tales como videos y aplicaciones informáticas, a fin de modelar estructuras moleculares, las cuales son de su agrado.

Las actividades de orientación planificadas a realizar con los estudiantes se mencionan a continuación:

- Conferencias de actualización política y económica de Cuba y del país sede donde se efectúa la olimpiada.

- Conferencia: «Panorama geográfico y social de los países».
- Conferencia: «Sistema político cubano».

- Talleres de reflexión metacognitiva.

Además, se tuvo en cuenta que algunas de estas actividades debían realizarlas estudiantes y profesores en conjunto, dada la importancia de la información y actualización cultural para ambos.

Una vez planificada las actividades, el colectivo de profesores entrenadores las discutió con el propósito de conocer las opiniones de los estudiantes y agregar algunas de su interés. A la par se conversó con algunos padres y se solicitó ayuda para la coordinación de actividades por fuera del centro de entrenamiento.

Luego de la planificación y consulta con todos los involucrados, se realizó un cronograma de actividades.

\subsection{Etapa 3}

Instrumentación y control de las acciones de estimulación y orientación para el desarrollo del talento académico en química, en las tres esferas: cognitiva-instrumental, motivacional-afectiva y psicosocial.

La tercera etapa del programa fue la instrumentación de las actividades de estimulación y orientación enfocadas hacia el desarrollo del talento académico en química, en las tres esferas: cognitiva-instrumental, motivacional-afectiva y psicosocial. 
El objetivo específico de esta etapa fue ejecutar las actividades planificadas.

El plan temático planificado se cumplió en el desarrollo de las clases en el centro de entrenamiento y las actividades de orientación en este y en los centros de investigación y recreativos.

Las conferencias de actualización política y económica de Cuba, y del país sede donde se efectúa la olimpiada, las impartió un Doctor en Ciencias en la especialidad de Geografía, de la Universidad de Ciencias Pedagógicas, así como la conferencia «Panorama geográfico y social de los países».

La conferencia "Sistema político cubano» fue impartida por el también Doctor en Ciencias y profesor entrenador, quien, además, era diputado a la Asamblea Nacional.

Los talleres de reflexión metacognitiva los realizaron en conjunto tres de los profesores entrenadores.

Las actividades de orientación que se planificaron realizar con los entrenadores fueron necesarias por cuanto son los mediadores del proceso de enseñanza-aprendizaje proceso de entrenamiento-y contribuyen a la preparación que requiere cada estudiante según sus necesidades y potencialidades. Por esta razón recibieron, al igual que los estudiantes, la conferencia de actualización política y económica de Cuba y del país sede donde se efectúa la olimpiada, pero en este caso se persiguió el objetivo de que conocieran los aspectos abordados con los estudiantes y estuvieran en capacidad de realizar el trabajo educativo que se requiere con estos.

Otra actividad de orientación dirigida a los entrenadores fue la información de los resultados obtenidos en la caracterización de cada estudiante de la Preselección Nacional, a fin de que, en el proceso de entrenamiento, los profesores incentivaran, potenciaran y desarrollaran la actividad independiente en la búsqueda y la construcción de nuevos conocimientos, la formación de valores y de sentimientos en estos estudiantes.

Con la familia de los estudiantes de la preselección se organizaron charlas de orientación sobre la atención que requieren los estudiantes con talento académico en química. Entre los aspectos que fueron objeto de análisis se encontraron: el desempeño cognitivo, el estilo de enfrentamiento al éxito y al fracaso, la motivación de logro, la tolerancia al estrés, el reconocimiento al logro, la satisfacción que siente en el colectivo estudiantil, la autoestima y la comunicación con los demás, así como los familiares recibieron recomendaciones de cómo proceder de acuerdo con las características de cada estudiante.

Además, se realizaron actividades extracurriculares que contribuyeron al desarrollo del talento académico en química, como, por ejemplo, la visita al Centro Nacional de Investigaciones Científicas, consultas en internet y la visita a actividades culturales y deportivas. 
3.4 Etapa 4. Evaluación del programa educativo para el desarrollo del talento académico en química de los estudiantes

Una vez ejecutadas las actividades fue necesario constatar el nivel alcanzado en la ejecución de cada una, lo que en su totalidad permitió la evaluación del programa educativo para el desarrollo del talento académico en química de los estudiantes.

Los objetivos específicos de esta etapa fueron:

- Valorar el cumplimiento de las actividades realizadas en función de la identificación, la estimulación y la orientación de los estudiantes.
- Trazar nuevas acciones que contribuyan al desarrollo del talento académico en química de los estudiantes, en una nueva etapa de entrenamiento.

En esta etapa fue necesario el empleo de pruebas pedagógicas y de técnicas de identificación como, por ejemplo, «¿Quién es el más?», y el test de satisfacción grupal.

Luego de la valoración de los resultados, se rediseñaron acciones en conjunto a partir de las opiniones recibidas de los profesores entrenadores, de los estudiantes y de sus padres.

\section{Resultados y discusión de la aplicación del programa educativo}

La factibilidad del programa educativo que se propuso se valoró a partir del desarrollo de un experimento pedagógico en el colectivo de los doce estudiantes que conformó la Preselección Nacional de Química - preexperimento-, con el objetivo de estudiar el comportamiento del programa en la práctica pedagógica, el cual tuvo como hipótesis: «El programa educativo contribuye al desarrollo del talento académico en química desde las esferas cognitiva-instrumental, motivacional-afectiva y psicosocial, en el proceso de entrenamiento de los estudiantes de la Preselección Nacional». 
A fin de comprobar la hipótesis del preexperimento se realizaron las acciones que se describen a continuación.

4.1 Diagnóstico del estado inicial del desarrollo del talento académico en química desde las esferas cognitivainstrumental, motivacional-afectiva y psicosocial, en el proceso de entrenamiento de los estudiantes que conformaban la Preselección Nacional

La prueba pedagógica aplicada la conformaron los profesores entrenadores y los contenidos evaluados fueron de varias asignaturas de química, acorde con los indicadores a medir en la investigación.

Los resultados obtenidos en la prueba pedagógica en el inicio del entrenamiento se reflejan en la tabla 4.

Tabla 4. Resultados de la prueba pedagógica inicial

\begin{tabular}{|c|c|c|c|c|c|c|c|c|c|c|c|c|c|}
\hline Indicador/estudiante & 1 & 2 & 3 & 4 & 5 & 6 & 7 & 8 & 9 & 10 & 11 & 12 & Md \\
\hline Nivel de conocimientos sobre química general e inorgánica & 3 & 5 & 5 & 4 & 5 & 5 & 4 & 5 & 5 & 5 & 5 & 4 & 4,75 \\
\hline Nivel de conocimientos sobre química física & 3 & 2 & 3 & 3 & 3 & 3 & 2 & 4 & 3 & 4 & 3 & 4 & 3,07 \\
\hline Nivel de conocimientos sobre química orgánica & 2 & 2 & 2 & 2 & 2 & 2 & 2 & 2 & 3 & 4 & 3 & 4 & 2,0 \\
\hline Nivel de conocimientos sobre química analítica & 4 & 4 & 5 & 2 & 5 & 4 & 5 & 3 & 3 & 5 & 4 & 3 & 4,0 \\
\hline $\begin{array}{l}\text { Nivel de desarrollo de habilidades con la nomenclatura } \\
\text { química }\end{array}$ & 5 & 5 & 5 & 5 & 5 & 5 & 5 & 5 & 5 & 5 & 5 & 5 & 5,0 \\
\hline Nivel de desarrollo de habilidades con la notación química & 5 & 5 & 5 & 5 & 5 & 5 & 5 & 5 & 5 & 5 & 5 & 5 & 5,0 \\
\hline $\begin{array}{l}\text { Nivel de desarrollo de habilidades con la escritura de } \\
\text { ecuaciones químicas }\end{array}$ & 3 & 5 & 5 & 5 & 5 & 5 & 5 & 5 & 5 & 5 & 5 & 3 & 4,9 \\
\hline $\begin{array}{l}\text { Nivel de desarrollo de habilidades con la interpretación de } \\
\text { gráficos }\end{array}$ & 4 & 1 & 2 & 3 & 4 & 4 & 4 & 3 & 4 & 4 & 4 & 4 & 3,75 \\
\hline $\begin{array}{l}\text { Nivel de desarrollo de habilidades con la interpretación de } \\
\text { tablas }\end{array}$ & 5 & 5 & 5 & 5 & 5 & 5 & 5 & 5 & 5 & 5 & 5 & 5 & 5,0 \\
\hline $\begin{array}{l}\text { Nivel de desarrollo de habilidades con la interpretación } \\
\text { cuantitativa de una ecuación química }\end{array}$ & 5 & 5 & 5 & 5 & 5 & 5 & 5 & 5 & 5 & 5 & 5 & 5 & 5,0 \\
\hline $\begin{array}{l}\text { Nivel de desarrollo de habilidades con la interpretación } \\
\text { cualitativa de una ecuación química }\end{array}$ & 5 & 5 & 5 & 5 & 5 & 5 & 5 & 5 & 5 & 5 & 5 & 5 & 5,0 \\
\hline
\end{tabular}




\begin{tabular}{|c|c|c|c|c|c|c|c|c|c|c|c|c|c|}
\hline $\begin{array}{l}\text { Nivel de desarrollo de habilidades con la resolución de } \\
\text { problemas químicos con cálculo }\end{array}$ & 2 & 5 & 5 & 2 & 5 & 1 & 2 & 1 & 5 & 5 & 2 & 5 & 2,5 \\
\hline $\begin{array}{l}\text { Nivel de desarrollo de habilidades con la interpretación } \\
\text { espacial de estructuras moleculares }\end{array}$ & 2 & 4 & 4 & 4 & 4 & 4 & 4 & 4 & 5 & 5 & 4 & 4 & 4,28 \\
\hline $\begin{array}{l}\text { Nivel de desarrollo de habilidades con el montaje de } \\
\text { aparatos de laboratorio }\end{array}$ & 1 & 1 & 1 & 1 & 1 & 1 & 1 & 1 & 4 & 5 & 4 & 5 & 1,25 \\
\hline $\begin{array}{l}\text { Nivel de desarrollo de habilidades con la manipulación de } \\
\text { la dotación química de laboratorio }\end{array}$ & 1 & 1 & 1 & 1 & 1 & 1 & 1 & 1 & 4 & 5 & 4 & 5 & 1,25 \\
\hline $\begin{array}{l}\text { Nivel de desarrollo de habilidades con la resolución de } \\
\text { problemas experimentales }\end{array}$ & 4 & 1 & 2 & 3 & 4 & 4 & 4 & 3 & 4 & 4 & 4 & 4 & 3,75 \\
\hline
\end{tabular}

Fuente. Elaboración propia.

La interpretación de los resultados de la tabla 4 permitió conocer los contenidos y las habilidades en las que cada estudiante estaba por debajo de la media aritmética de cada indicador.

Los resultados, en atención a los instrumentos test de autoconcepto, inventario de ansiedades, test de satisfacción grupal, inventario de autoestima, la composición y la entrevista personal estructurada aplicados, reflejaron que el comportamiento de las dimensiones motivacional-afectiva y psicosocial tienen una tendencia de alto, al igual que la dimensión psicosocial.

En el análisis de la coherencia entre los indicadores se aplicó el coeficiente de correlación multidimensional $\left(r_{p j}\right)$ para determinar el grado de coherencia que existe entre los indicadores que las caracterizaron, a partir de los rangos tipificados que se muestran en la tabla 5.

Tabla 5. Rangos tipificados

\begin{tabular}{|c|c|}
\hline Valores de $r_{P j}$ & Grado de coherencia \\
\hline $1>r_{P j}>0,9$ & Muy alta coherencia \\
\hline $0,89>r_{P j}>0,8$ & Alta coherencia \\
\hline $0,79>r_{P j}>0,7$ & Coherencia \\
\hline $0,69>r_{P j}>0,6$ & Alguna coherencia \\
\hline $0,59>r_{P j}>0$ & Poca coherencia \\
\hline
\end{tabular}

Fuente. Elaboración propia. 
Los resultados de la aplicación del coeficiente de correlación multidimensional $\left(r_{p j}\right)$ al sistema de indicadores en la etapa inicial se muestran en la tabla 6.

Tabla 6. Resultado del coeficiente $\left(r_{P j}\right)$

\begin{tabular}{|c|c|}
\hline Dimensiones & $r_{P j}$ \\
\hline Cognitiva-instrumental & 0,65 \\
\hline Motivacional-afectiva & 0,55 \\
\hline Psicosocial & 0,57 \\
\hline
\end{tabular}

Fuente. Elaboración propia.

Al contrastar los resultados obtenidos con la tabla que tipifica los diferentes niveles de coherencia se constata que estos niveles se encuentran muy por debajo de los límites establecidos para considerarlos confiables en razón al bajo grado de coherencia racional.

Los resultados obtenidos por los estudiantes en la dimensión cognitiva-instrumental indicaron que existían insuficiencias en el dominio de los contenidos relacionados con el nivel de conocimientos sobre química orgánica, química física y química analítica, necesarios para competir en las olimpiadas. Esto se acentúa en el nivel de conocimientos sobre química orgánica, en el nivel de desarrollo de las habilidades relacionadas con la manipulación de la dotación de laboratorio y en el montaje de aparatos. Lo anterior indica carencias en los estudiantes para interrelacionar los contenidos $y$, por tanto, la imposibilidad de utilizarlos de manera eficiente en su práctica.
En las dimensiones motivacional-afectiva y psicosocial se revela una alta autovaloración de la labor que realizan, así como un alto autoconcepto académico y social. Sin embargo, algunos procesos psíquicos distorsionan la posibilidad de que los estudiantes posean altos desempeños, ya que manifiestan una tendencia a un autoconcepto afectivo bajo al sentir temor al fracaso o a no cumplir las expectativas de sus padres, quienes en ocasiones presionan a sus hijos en busca de un mayor resultado sin tener en cuenta el apoyo psicológico que deben darles, bien sea por desconocimiento o bien por características propias de su personalidad. Este aspecto fue declarado por los estudiantes en entrevistas realizadas. Además, algunos de ellos poseen un nivel medio de autoestima y un nivel alto de ansiedad, lo que los lleva a obtener resultados por debajo de su posible rendimiento académico. 


\subsection{Aplicación de la prueba pedagógica final}

La prueba pedagógica final la elaboraron los profesores entrenadores. La aplicaron luego de la culminación del periodo lectivo en el que se cumplió el plan temático planificado y contempla los indicadores establecidos para la investigación.

Los resultados de la prueba pedagógica final se reflejan en la tabla 7.

Tabla 7. Resultados de la prueba pedagógica final

\begin{tabular}{|c|c|c|c|c|c|c|c|c|c|c|c|c|c|}
\hline Indicador & 1 & 2 & 3 & 4 & 5 & 6 & 7 & 8 & 9 & 10 & 11 & 12 & Md \\
\hline $\begin{array}{l}\text { Conocimientos sobre química general e } \\
\text { inorgánica }\end{array}$ & 5 & 5 & 5 & 4 & 5 & 5 & 5 & 5 & 5 & 5 & 5 & 5 & 4,95 \\
\hline Conocimientos sobre química física & 3 & 3 & 4 & 4 & 3 & 4 & 4 & 5 & 4 & 5 & 3 & 4 & 3.83 \\
\hline Conocimientos sobre química orgánica & 3 & 3 & 4 & 3 & 4 & 4 & 4 & 4 & 4 & 5 & 3 & 4 & 3,79 \\
\hline Conocimientos sobre química analítica & 4 & 4 & 5 & 3 & 4 & 3 & 5 & 4 & 4 & 5 & 4 & 4 & 4,07 \\
\hline $\begin{array}{l}\text { Desarrollo de habilidades en la } \\
\text { nomenclatura química }\end{array}$ & 5 & 5 & 5 & 5 & 5 & 5 & 5 & 5 & 5 & 5 & 5 & 5 & 5,0 \\
\hline $\begin{array}{l}\text { Desarrollo de habilidades en la notación } \\
\text { química }\end{array}$ & 5 & 5 & 5 & 5 & 5 & 5 & 5 & 5 & 5 & 5 & 5 & 5 & 5,0 \\
\hline $\begin{array}{l}\text { Desarrollo de habilidades en la escritura } \\
\text { de ecuaciones químicas }\end{array}$ & 5 & 5 & 5 & 5 & 5 & 5 & 5 & 5 & 5 & 5 & 5 & 5 & 5,0 \\
\hline $\begin{array}{l}\text { Desarrollo de habilidades en la } \\
\text { interpretación de gráficos }\end{array}$ & 4 & 3 & 4 & 3 & 4 & 5 & 5 & 5 & 5 & 5 & 5 & 5 & 4,64 \\
\hline $\begin{array}{l}\text { Desarrollo de habilidades en la } \\
\text { interpretación de tablas }\end{array}$ & 5 & 5 & 5 & 5 & 5 & 5 & 5 & 5 & 5 & 5 & 5 & 5 & 5,0 \\
\hline $\begin{array}{l}\text { Desarrollo de habilidades en la } \\
\text { interpretación cuantitativa de una ecuación } \\
\text { química }\end{array}$ & 5 & 5 & 5 & 5 & 5 & 5 & 5 & 5 & 5 & 5 & 5 & 5 & 5,0 \\
\hline $\begin{array}{l}\text { Desarrollo de habilidades en la } \\
\text { interpretación cualitativa de una ecuación } \\
\text { química }\end{array}$ & 5 & 5 & 5 & 5 & 5 & 5 & 5 & 5 & 5 & 5 & 5 & 5 & 5,0 \\
\hline
\end{tabular}




\begin{tabular}{|c|c|c|c|c|c|c|c|c|c|c|c|c|c|}
\hline $\begin{array}{l}\text { Desarrollo de habilidades en la resolución } \\
\text { de problemas químicos con cálculo }\end{array}$ & 4 & 4 & 5 & 3 & 4 & 3 & 5 & 4 & 4 & 5 & 4 & 4 & 4,07 \\
\hline $\begin{array}{l}\text { Desarrollo de habilidades en la } \\
\text { interpretación espacial de estructuras } \\
\text { moleculares }\end{array}$ & 3 & 4 & 5 & 3 & 4 & 4 & 5 & 5 & 4 & 5 & 4 & 4 & 4,17 \\
\hline $\begin{array}{l}\text { Desarrollo de habilidades en el montaje de } \\
\text { aparatos de laboratorio }\end{array}$ & 4 & 4 & 5 & 4 & 4 & 4 & 4 & 5 & 5 & 5 & 5 & 5 & 4,5 \\
\hline $\begin{array}{l}\text { Desarrollo de habilidades en la } \\
\text { manipulación de la dotación química de } \\
\text { laboratorio }\end{array}$ & 3 & 4 & 4 & 4 & 4 & 4 & 4 & 5 & 5 & 5 & 5 & 5 & 4,33 \\
\hline $\begin{array}{l}\text { Desarrollo de habilidades en la resolución } \\
\text { de problemas experimentales }\end{array}$ & 4 & 3 & 5 & 3 & 4 & 4 & 5 & 5 & 4 & 5 & 5 & 5 & 4,5 \\
\hline
\end{tabular}

Fuente. Elaboración propia.

Del análisis de la comparación del resultado alcanzado en cada indicador en la prueba inicial y final se determinó que no se produjeron cambios significativos en los indicadores nivel de desarrollo de habilidades con la nomenclatura química, la notación química, la escritura de ecuaciones, la interpretación de tablas y la interpretación cualitativa y cuantitativa de las ecuaciones químicas, ya que en el estado inicial todos los integrantes del grupo de estudio alcanzaron la categoría de muy alta. En el resto de los indicadores de la dimensión dominio de los contenidos químicos se apreció un cambio significativo en los resultados obtenidos.

La dimensión estado de los procesos psíquicos cognitivos se controló mediante el método de observación por los profesores entrenadores, al igual que las dimensiones motivacional-afectiva y psicosocial. Los resultados obtenidos se registraron y los presenta la tabla 8.

Tabla 8. Resultados de las observaciones registradas

\begin{tabular}{|l|c|c|c|c|c|c|c|c|c|c|c|c|c|}
\hline \multicolumn{1}{|c|}{ Indicador } & $\mathbf{1}$ & $\mathbf{2}$ & $\mathbf{3}$ & $\mathbf{4}$ & $\mathbf{5}$ & $\mathbf{6}$ & $\mathbf{7}$ & $\mathbf{8}$ & $\mathbf{9}$ & $\mathbf{1 0}$ & $\mathbf{1 1}$ & $\mathbf{1 2}$ & $\mathbf{M d}$ \\
\hline Razonamiento & 5 & 4 & 5 & 4 & 5 & 5 & 5 & 5 & 5 & 5 & 5 & 5 & 4,9 \\
\hline Imaginación & 5 & 5 & 5 & 5 & 5 & 5 & 5 & 5 & 5 & 5 & 5 & 5 & 5,0 \\
\hline Memoria & 5 & 5 & 5 & 5 & 5 & 5 & 5 & 5 & 5 & 5 & 5 & 5 & 5,0 \\
\hline Autoconcepto académico & 4 & 4 & 5 & 4 & 4 & 4 & 5 & 5 & 5 & 5 & 5 & 5 & 4,64 \\
\hline
\end{tabular}




\begin{tabular}{|c|c|c|c|c|c|c|c|c|c|c|c|c|c|}
\hline Autoconcepto afectivo & 4 & 4 & 5 & 4 & 4 & 4 & 4 & 5 & 5 & 5 & 5 & 5 & 4,5 \\
\hline Autoconcepto social & 5 & 4 & 5 & 5 & 5 & 4 & 4 & 5 & 5 & 5 & 5 & 5 & 4,83 \\
\hline Apoyo familiar & 5 & 5 & 5 & 3 & 4 & 5 & 5 & 5 & 5 & 5 & 5 & 5 & 5,0 \\
\hline Ansiedad & 2 & 2 & 1 & 2 & 2 & 2 & 1 & 1 & 1 & 1 & 1 & 1 & 1,36 \\
\hline Satisfacción grupal & 5 & 5 & 5 & 5 & 5 & 5 & 5 & 5 & 5 & 5 & 5 & 5 & 5,0 \\
\hline Autoestima & 4 & 4 & 5 & 4 & 4 & 4 & 5 & 5 & 5 & 5 & 5 & 5 & 4,64 \\
\hline
\end{tabular}

Fuente. Elaboración propia.

Una vez se procesó la información que se presenta en la tabla 8 se procedió a realizar un análisis de los resultados alcanzados por cada estudiante $y$, de forma general, lo que se representa de forma gráfica en las figuras 1, 2, 3 y 4 .

Figura 1. Comparación del estado inicial y final de los indicadores de las subdimensiones contenidos químicos y desarrollo de habilidades

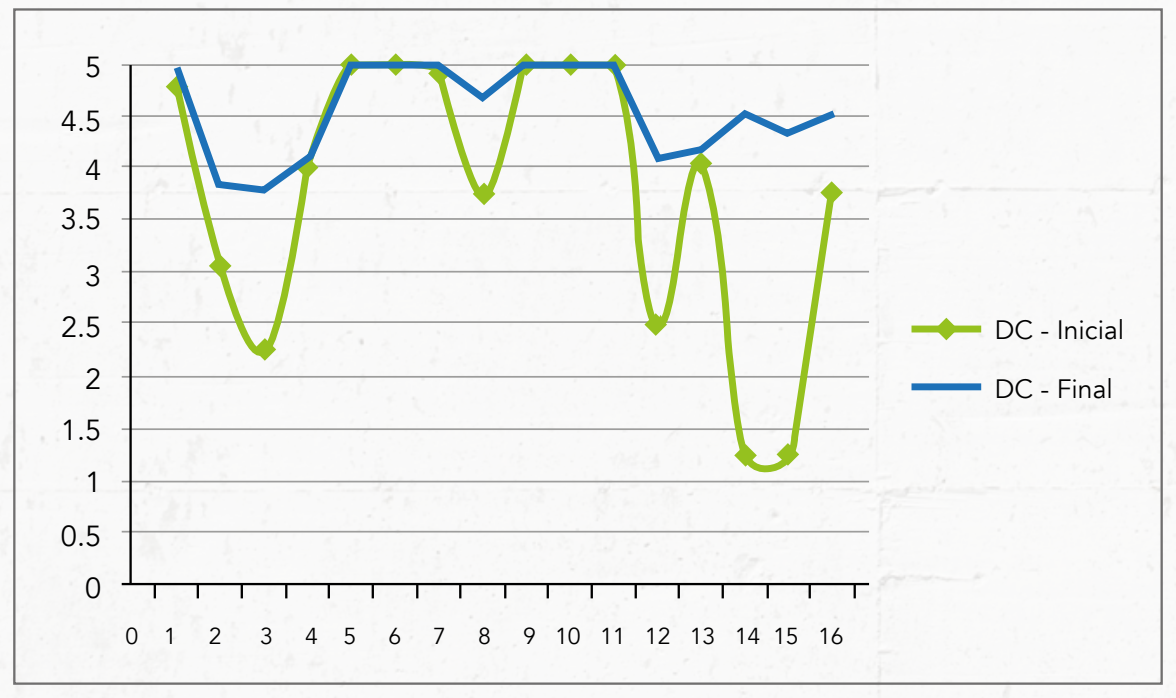

Fuente. Elaboración propia. 
Figura 2. Comparación del estado inicial y final de la subdimensión estado de los procesos psíquicos cognitivos

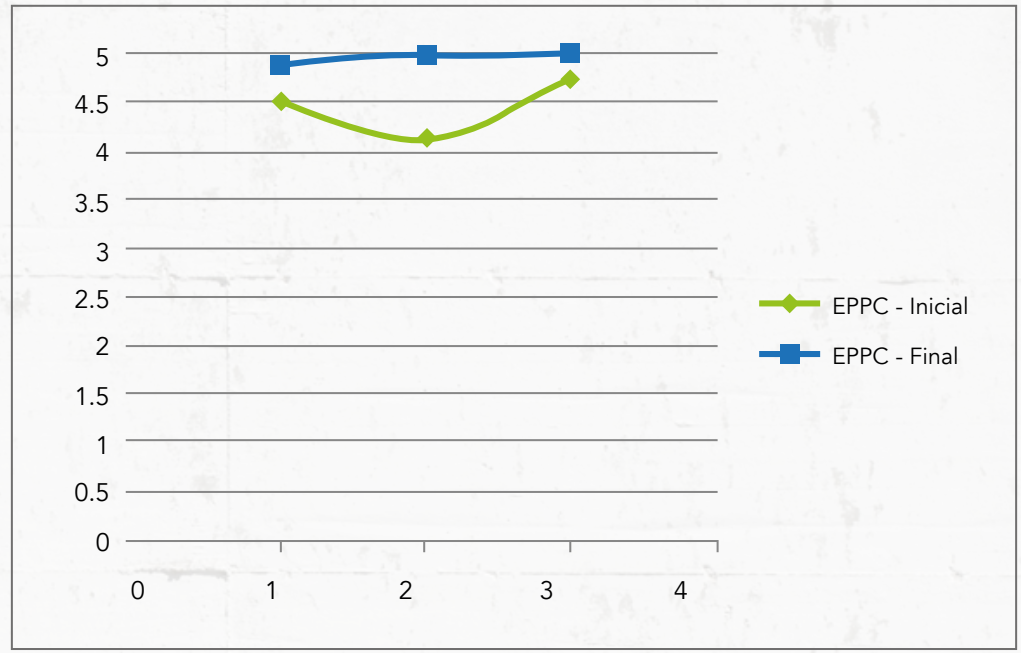

Fuente. Elaboración propia.

Figura 3. Comparación del estado inicial y final de la dimensión motivacional-afectiva

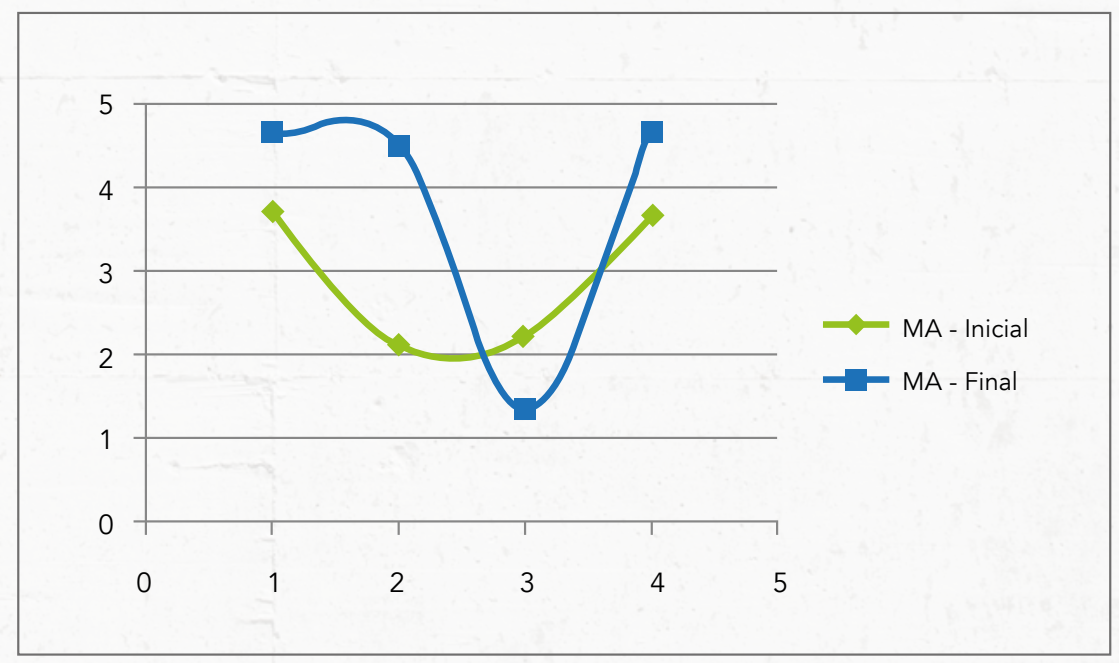

Fuente. Elaboración propia. 
Figura 4. Comparación del estado inicial y final de la dimensión psicosocial

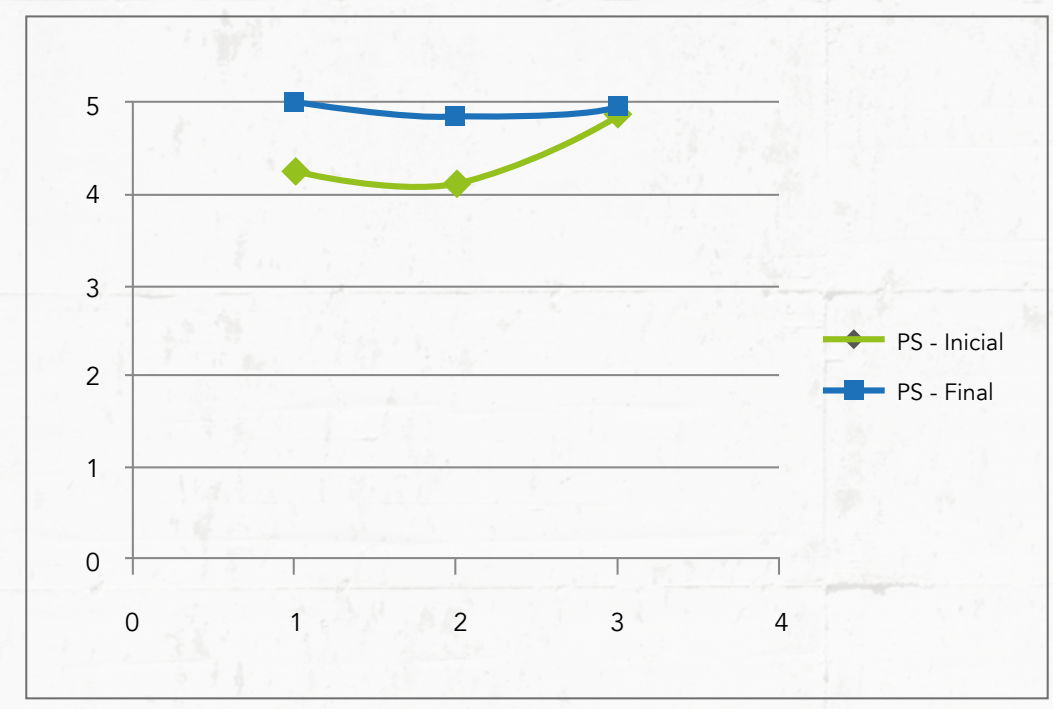

Fuente. Elaboración propia.

El análisis de las figuras 1, 2, 3, y 4 destaca que en la subdimensión dominio del contenido, de la dimensión cognitiva-instrumental, los indicadores nivel de conocimientos sobre química física y química orgánica evidencian la mayor transformación en los estudiantes, pues en estos indicadores los cambios más significativos se produjeron en cinco estudiantes y los cambios menos significativos en otros cinco, y el resto del grupo mantiene el nivel constatado antes de desarrollar el programa educativo; esto refleja una tendencia al mejoramiento del nivel de conocimientos de estas asignaturas.
Otro aspecto a destacar es que en la subdimensión desarrollo de habilidades químicas, de la dimensión cognitivainstrumental, los indicadores nivel de desarrollo de habilidades en el montaje de aparatos y manipulación de la dotación química de laboratorio evidencian la mayor transformación en los estudiantes, ya que los cambios más significativos se produjeron en ocho estudiantes y los cambios menos significativos en dos. Esto refleja una tendencia superior en el nivel de desarrollo de estas habilidades y se destaca que, de modo general, se ha logrado que todos los estudiantes del grupo de estudio alcancen un alto nivel en estos indicadores. 
En la dimensión motivacional-afectiva, la mayor transformación en los estudiantes se evidenció en los indicadores autoconcepto afectivo. En estos, nueve estudiantes alcanzaron la evaluación de muy alto, ya que disminuyeron en gran medida sus temores al fracaso al alcanzar mayor seguridad en sí mismos luego de desarrollar las acciones del programa educativo.

En la dimensión psicosocial, en el indicador autoconcepto social, se observó la mayor transformación en tres de los estudiantes al final de la aplicación del programa educativo: de forma general, todos los integrantes del grupo de estudio muestran una tendencia a establecer una alta comunicación con los demás.

A fin de valorar las transformaciones producidas en los doce estudiantes en las dimensiones cognitiva-instrumental, motivacional-afectiva y psicosocial se aplicó el coeficiente de correlación multidimensional $\left(r_{p j}\right)$ a los resultados de cada indicador, después de aplicado el programa educativo.

Los resultados alcanzados en el inicio y en el final se muestran en la tabla 9.

Tabla 9. Resultados del coeficiente $\left(r_{p j}\right)$ en el inicio y en el final de la investigación

\begin{tabular}{|c|c|c|}
\hline \multirow{2}{*}{ Dimensiones } & \multicolumn{2}{|c|}{$r_{\mathrm{pj}}$} \\
\cline { 2 - 3 } & Inicial & Final \\
\hline Cognitiva-instrumental & 0,65 & 0,73 \\
\hline Motivacional-afectiva & 0,55 & 0,97 \\
\hline Psicosocial & 0,57 & 0,70 \\
\hline
\end{tabular}

Fuente. Elaboración propia.

Los resultados comparativos del estado inicial y final muestran una mayor coherencia racional en los indicadores de las dimensiones objeto de investigación. En la dimensión cognitiva-instrumental, en el inicio, existía alguna coherencia entre los indicadores, y en el final existió coherencia entre ellos; en la dimensión motivacional-afectiva de poca coherencia en el inicio se obtuvo muy alta coherencia entre sus indicadores en el final, y en la dimensión psicosocial de poca coherencia en el inicio se obtuvo coherencia entre los indicadores en el final. Esto evidencia que los estudiantes están mejor preparados para manifestar sus potencialidades talentosas en química. 


\section{Conclusiones}

A continuación, se presentan las conclusiones de la etapa de diagnóstico del desarrollo del talento académico en química de los estudiantes, en las tres dimensiones de la investigación.

En la dimensión cognitiva-instrumental se detectaron insuficiencias en el dominio de los contenidos relacionados con el nivel de conocimiento en química orgánica, química física y química analítica. Además, en el desarrollo de habilidades en la manipulación de la dotación de laboratorio y en el montaje de aparatos, lo que denotó la carencia en la interrelación de los contenidos y la imposibilidad de utilizarlos de manera eficiente en la práctica.

En las dimensiones motivacional-afectiva y psicosocial se comprobó que los estudiantes poseían una alta valoración de la labor que realizaban, pero algunos procesos psíquicos distorsionaban la posibilidad de que ellos se desempeñaran con un alto rendimiento.

En las etapas de planificación y ejecución de las acciones del programa educativo participaron, además de los estudiantes de la preselección nacional, los profesores entrenadores, los familiares de los estudiantes y algunos profesionales de universidades y de centros de investigaciones, quienes - de forma conjunta- formaron un equipo de trabajo en el que predominó la colaboración y el apoyo.
En la etapa de evaluación de la factibilidad del programa educativo se emplearon técnicas de investigación que permitieron evaluar los indicadores en la etapa final del entrenamiento de los estudiantes en las tres dimensiones. Además, se aplicó el coeficiente de correlación multidimensional, a fin de valorar la coherencia racional en los indicadores de la variable. Esto permitió conocer cómo en el inicio de la investigación, en la dimensión cognitiva instrumental, existía alguna coherencia entre los indicadores, y en el final existió coherencia entre ellos. Por su parte, en las dimensiones motivacional-afectiva y psicosocial, en el inicio existió muy poca coherencia $y$, en el final, en la primera una muy alta coherencia, mientras en la segunda una coherencia entre los indicadores. Esto evidenció transformaciones en los modos de actuación de los estudiantes.

Los familiares y los profesores entrenadores manifestaron su conformidad con las acciones realizadas y colaboraron en su diseño para el nuevo periodo de entrenamiento. La valoración de los resultados, de forma general, corroboró que el programa educativo para el desarrollo del talento académico en química es factible. También es necesario apuntar que en su aplicación se deben tener en cuenta las necesidades y las potencialidades de los estudiantes que conformen la Preselección Nacional, con el objetivo de ajustar, si es necesario, las acciones de este programa. Además, estos deben prepararse desde las esferas cognitivainstrumental, motivacional-afectiva y psicosocial, con el fin de lograr una preparación integral que les permita enfrentar los retos futuros de la sociedad. 


\section{Referencias}

Castellanos, D. (1997). Modelo heurístico para la identificación del talento en el contexto escolar. La Habana: ISP «Enrique José Varona».

Castellanos, D. (2003). Talento: estrategias para su desarrollo. La Habana: Pueblo y Educación.

Catellanos, D. (2005). La educación y el desarrollo del talento. La Habana: Pueblo y Educación.

Delgado, N. (2004). La olimpiada de química. Una propuesta de estrategia. La Habana: IV Congreso Internacional Didáctica de las Ciencias.

Delgado, N. (2010). Programa educativo para el desarrollo del talento químico de los estudiantes de la preselección nacional. La Habana: ISP «Enrique José Varona». 